\title{
THERMAL-VACUUM RESPONSE OF POLYMER MATRIX COMPOSITES IN SPACE
}

\author{
R.C. Tennyson and R. Matthews \\ University of Toronto Institute for Aerospace Studies \\ North York, Ontario, Canada, M3H 5T6 \\ Phone: 416/667-7710, Fax: 416/667-7799
}

\section{SUMMARY}

This report describes a thermal-vacuum outgassing model and test protocol for predicting outgassing times and dimensional changes for polymer matrix composites. Experimental results derived from "control" samples are used to provide the basis for analytical predictions to compare with the outgassing response of Long Duration Exposure Facility (LDEF) flight samples. Coefficient of thermal expansion (CTE) data are also presented. In addition, an example is given illustrating the dimensional change of a "zero" CTE laminate due to moisture outgassing.

\section{THERMAL-VACUUM OUTGASSING AND DIMENSIONAL CHANGES OF LDEF POLYMER MATRIX COMPOSITES (AO180)}

The University of Toronto Institute for Aerospace Studies (UTIAS) experiment consisted of a variety of graphite, aramid, and boron fiber reinforced epoxy matrix composites located at station D12 on LDEF (i.e., $\sim 82^{\circ}$ relative to velocity vector). Selected samples were instrumented with strain and temperature gauges that were sampled every 16 hours over the first 370 days in orbit. Data were stored on a magnetic tape cassette using a space-qualified data acquisition system designed and constructed at UTIAS. Details on this aspect of our experiment can be obtained from reference 1 . It was found that the strain/thermal gauge measuring system worked flawlessly, as evidenced by the measured response of a stainless steel calibration specimen which remained unchanged throughout the 5.75 years in orbit. Typical time/temperature and strain/temperature data for one material (graphite/epoxy, 5208/T300) are shown in Figures 1 and 2, respectively. This data can be replotted as strain versus temperature as given in Figure 3 for the $90^{\circ}$ laminate. It can be seen that a "total" dimensional strain change of $\sim 1,600 \times 10^{-6}$ occurred after about 80 days in orbit. It should be noted that no microcracks were observed in this laminate, and full recovery of the dimensional change resulted once the sample was returned to Earth and exposed to the ambient environment.

From these data, it is possible to estimate the CTE from the final slope once all outgassing is essentially finished. Using this CTE value, one can correct for the temperature variations on-orbit, giving the strain change of the sample, over time, independent of temperature. The formula used to do this is:

$$
\Delta_{t}=\varepsilon_{t}-\left(T_{t}-T_{\mathrm{Ref}}\right) \cdot \alpha
$$

where $\Delta_{t}=$ strain change at time $t, \varepsilon_{t}=$ measured strain at time $t, T_{t}=$ temperature at time $t$, $T_{\text {Ref }}=$ reference temperature $=75^{\circ} \mathrm{F}$, and $\alpha=$ CTE of material. 
$\Delta_{t}$ was then plotted against time and an adjustment factor ( $\Delta$ adj) was added to every point. This had the effect of shifting the graph so that the final strain was zero, allowing the total strain change to be read easily. Figure 4 shows the adjusted $\Delta_{t}$ versus time curve for a $90^{\circ}$ graphite/epoxy laminate $(5208 / \mathrm{T} 300)$. From this graph it is evident that outgassing was completed in about 80 to 100 days. It is clear that outgassing was very rapid over the first 25 days, then slowed due to the low temperatures encountered (Fig. 1). Outgassing then increased after 50 days as the sample temperature increased, and eventually no further measured dimensional change occurred after about 80 to 100 days exposure. Similar behavior was exhibited by the other composite materials (ref. 1). It is interesting to note that in the fiber direction (i.e., a $0^{\circ}$ laminate), very small $\Delta_{t}$ changes were observed, as illustrated in Figure 5 for another graphite/epoxy material (SP288/T300). In general, the outgassing time required to reach an equilibrium state in space depends on such factors as the initial moisture concentration, the volatile content, laminate thickness, ambient temperature, and constituent material diffusion properties.

\section{MOISTURE DESORPTION AND DIMENSIONAL CHANGES}

As with many other published analyses, the moisture desorption $M$ can be estimated using Fick's law from the equation (see, for example, ref. 2),

$$
M(t)_{T=\mathrm{const}} \cong M_{O} \exp \left[-7.3\left(\frac{D t}{h^{2}}\right)^{0.75}\right]
$$

where $M_{o}=$ initial moisture content, $D=$ diffusion coefficient, and $h=$ thickness. For constant temperature, Shen and Springer (ref. 3) have shown that the diffusion coefficient $(D)$ can be calculated knowing the moisture content at different times from the relation,

$$
D_{T=\text { const }}=\frac{\pi h^{2}}{16 M_{o}^{2}}\left[\frac{M_{2}-M_{1}}{\sqrt{t_{2}}-\sqrt{t_{1}}}\right]^{2}
$$

where $M_{1}, M_{2}=$ moisture contents at times $t_{1}$ and $t_{2}$, respectively. that

Rather than measure moisture content during a test, one can employ strain data $(\varepsilon)$. Noting

$$
\varepsilon=M \beta
$$

where $\beta=$ coefficient of moisture expansion (CME), then equations (2) and (3) can be rewritten as,

and

$$
\varepsilon(t)_{T=\mathrm{const}}=\varepsilon_{o} \exp \left[-7.3\left(\frac{D t}{h^{2}}\right)^{0.75}\right]
$$

$$
D(t)_{T=\text { const }}=\frac{\pi h^{2}}{16 \varepsilon_{o}^{2}}\left[\frac{\varepsilon_{2}-\varepsilon_{1}}{\sqrt{t_{2}}-\sqrt{t_{1}}}\right]^{2} \text {. }
$$

To develop a model for predicting outgassing of materials in space, it is necessary to take temperature into account. However, Fick's law as previously described applies to constant temperature, constant humidity environments. In space, the humidity level (i.e., vacuum) is constant. 
Furthermore it is possible to determine a diffusion coefficient as a function of temperature $(D(t))$ by performing outgassing tests at different temperatures $\left(T_{a}\right.$ and $\left.T_{b}\right)$ assuming an Arrhenius relation between $D$ and $T$. This yields the equation,

$$
D(T)=\exp \left[\frac{\ln \left(D_{b}\right)-\ln \left(D_{a}\right)}{1-\frac{T_{a}}{T_{b}}}+\ln \left(D_{a}\right)\right] \cdot \exp \left[\frac{\left(\ln \left(D_{b}\right)-\ln \left(D_{a}\right)\right)}{\left(\frac{1}{T_{a}}-\frac{1}{T_{b}}\right) \cdot T}\right]
$$

This equation can be used to calculate the diffusion coefficient at any temperature, $T$, as long as the diffusion coefficients $D_{a}$ and $D_{b}$ at temperatures $T_{a}$ and $T_{b}$ are known. All of the above temperatures must be absolute $(\mathrm{K})$.

Hence, knowing $D(T)$, the strain associated with outgassing $\varepsilon(T, t)$ can be calculated from equation (5).

\section{LAMINATE ANALYSIS}

Consider an $N$-ply laminate characterized by a set of lamina properties defined by

$$
\phi_{l}=\left[\begin{array}{c}
\phi_{1} \\
\phi_{2} \\
0
\end{array}\right]
$$

where $\phi_{1}, \phi_{2}$ correspond to the fiber (1) and transverse (2) properties, respectively. Examples of $\phi_{m}$ include both CTE and CME coefficients, i.e.,

$$
\phi_{l}=\alpha_{l}=\left[\begin{array}{c}
\alpha_{1} \\
\alpha_{2} \\
0
\end{array}\right] \quad \text { and } \quad \phi_{l}=\beta_{l}=\left[\begin{array}{c}
\beta_{1} \\
\beta_{2} \\
0
\end{array}\right]
$$

where $\alpha=\varepsilon / \Delta T$ and $\beta=\varepsilon / \Delta M$. For an actual $N$-ply laminate consisting of a set of plies $k=1$ to $N$ having arbitrary orientations $\theta_{k}$ and stacking sequence, the structural properties defined by $\phi_{S}$ can be calculated from the following matrix equation (see ref. 4 for example):

$$
\left[\begin{array}{c}
\phi_{S} \\
\kappa_{T}
\end{array}\right]=\left[\begin{array}{cc}
A & B \\
B & D
\end{array}\right]^{-1}\left[\begin{array}{c}
J \\
H
\end{array}\right]\left[\begin{array}{c}
\phi_{1} \\
\phi_{2} \\
0
\end{array}\right]
$$

where

$$
\phi_{S}=\left[\begin{array}{c}
\phi_{x} \\
\phi_{y} \\
\phi_{x y}
\end{array}\right\rfloor, \quad \kappa_{T}=\left[\begin{array}{c}
\kappa_{x} \\
\kappa_{y} \\
\kappa_{x y}
\end{array}\right\rfloor=\text { laminate curvatures , }
$$




$$
\begin{aligned}
& \left|\begin{array}{ll}
A & B \\
B & D
\end{array}\right|^{-1}=\text { inverse of the standard laminate stiffness matrix (see ref. 4), } \\
& {[J]=\sum_{k=1}^{N} T_{k}^{-1} \cdot Q_{k}\left(h_{k}-h_{k-1}\right) \quad \text { and } \quad[H]=\sum_{k=1}^{N} \frac{1}{2} \cdot T_{k}^{-1} \cdot Q_{k}\left(h_{k}^{2}-h_{k-1}^{2}\right)} \\
& {[T]=\left|\begin{array}{ccc}
m^{2} & n^{2} & 2 m n \\
n^{2} & m^{2} & -2 m n \\
-m n & m n & m^{2}-n^{2}
\end{array}\right|=\text { transformation matrix , }} \\
& \quad Q_{k}=\text { reduced lamina stiffness matrix for } k \text { th ply (see ref. 4) } \\
& m=\cos \theta, \quad n=\sin \theta, \quad \theta=\text { ply angle, } \quad h_{k}=\text { thickness of } k \text { th ply. }
\end{aligned}
$$

\section{APPLICATION OF OUTGASSING DIMENSIONAL CHANGE ANALYSIS TO LDEF SAMPLES}

Prior to analyzing the LDEF data in detail, two issues regarding material response and the measuring systems warrant some discussion. The LDEF flight samples were monitored using bonded suriace strain gauges whereas the laboratory tests were conducted using laser interferometry. A comparison of both system responses is shown in Figure 6 where it is evident that excellent correlation exists based on the test of a flight sample in the vacuum chamber. The question of whether Fick's law is a good model for the graphite/epoxy composite material is addressed in Figure 7. Using a control sample that was vacuum dried and saturated to 0.49 -percent moisture content, then allowed to outgas at $\mathrm{T}=22{ }^{\circ} \mathrm{C}$, provided the $\varepsilon(t)$ curve shown. Employing the previous analysis to estimate the diffusion coefficient $D$, Fick's law prediction was compared to the measured long term response. Excellent agreement was obtained. Thus one can proceed with confidence in the analytical model and test procedures.

\section{EXPERIMENTAL INPUT}

The following test protocol was established utilizing LDEF "control" and "flight" samples. It should be noted that all "control" specimens were made at the same time from the same material batch as the flight articles, and stored at "ambient" laboratory conditions.

1. Samples were subjected to vacuum outgassing at elevated temperature to obtain their "dry weight" values.

2. For given temperature $(T)$ and percent $\mathrm{RH}$, moisture uptake (percent) was recorded for a given material from its "dry" state as a function of time $(t)$ to saturation. Figure 8 shows moisture absorption data for LDEF flight and control specimens (see Table 1).

3. Sample strain $(\varepsilon)$ was measured as a function of time $(t)$ in vacuum for two temperatures ( $T_{a}$ and $T_{b}$ ). Both experiments employed samples having the same $M_{o}$. 
Figures 9 to 12 present initial outgassing data for flight (2T13) and control (5T5) samples at temperatures of $22{ }^{\circ} \mathrm{C}$ and $55^{\circ} \mathrm{C}$. The strain response was measured in situ using laser interferometry.

\section{ANALYTICAL PROCEDURE}

1. Using the $\varepsilon(T, t)$ curves, the initial slope can be calculated from equation (7) to obtain $D_{a}\left(T_{a}\right)$ and $D_{b}\left(T_{b}\right)$.

2. Determine $D(T)$ from equation (7) based on $D_{a}\left(T_{a}\right), D_{b}\left(T_{b}\right), T_{a}$ and $T_{b}$. Table 1 summarizes the values obtained for $D(T)$ for both "flight" and "control" samples of graphite/epoxy $\left[90^{\circ}\right]_{4}$ laminates $(5208 / \mathrm{T} 300)$.

3. Using the LDEF temperature/time profile obtained in-orbit (Fig. 1), calculate the dynamic strain change $\varepsilon(t)$ for given time steps $(\Delta t)$, using the above $D(T)$ equation evaluated at the appropriate temperature. The $\varepsilon(t)$ function is given by (equation (5)),

$$
\varepsilon_{t}=\varepsilon_{t-1}-\varepsilon_{t-1}\left(1-\exp \left[-7.3\left(\frac{D\left(T_{t}\right) \cdot \Delta t}{h^{2}}\right)^{0.75}\right]\right),
$$

where $T_{t}=$ average temperature over $\Delta t$, assuming $\varepsilon_{o}$ is known at $t=0$ from the outgassing test. By using this equation at every time step over the temperature history, it is possible to calculate the strain change of the sample due to outgassing, taking into account temperature effects.

From the outgassing response shown in Figure 4, it is evident that the moisture diffusion process essentially ceases when the temperature drops to freezing or below (i.e., $D \approx 0$ when $T \leq$ $32^{\circ} \mathrm{F}$ ). This constraint can then be included in the $\varepsilon(t)$ prediction.

\section{COMPARISON WITH LDEF DATA}

Based on the data in Table 1, values of $D_{a} \cong 0.00013\left(\mathrm{~mm}^{2} / \mathrm{h}\right)$ and $D_{b} \simeq 0.00078\left(\mathrm{~mm}^{2} / \mathrm{h}\right)$ were selected, corresponding to temperatures of $22{ }^{\circ} \mathrm{C}$ and $50^{\circ} \mathrm{C}$, respectively. Using these results in equation (7) together with the temperature/time profile shown in Figure 1, the predicted dimensional change for the graphite/epoxy $90^{\circ}$ laminate (5208/T300) is plotted in Figure 13 with the measured LDEF response as a function of time in orbit. Curve 1 represents the case when no correction is applied for T $\leq 273 \mathrm{~K}$. One can see the effect of assuming "zero" outgassing of water moisture exhibited by curve 2 . Although the initial response prediction agrees well with flight data, it is clear that the predicted times to complete outgassing differ significantly from the flight measurements.

Theoretical predictions were then made for various values of the diffusion coefficients and the dimensional changes plotted in Figure 14. One can see in Figure 15 that for a diffusion coefficient of $D^{*} \simeq 0.134 D_{m}$ (where $D_{m}=$ measured value in the vacuum chamber), excellent agreement with the flight data is obtained. Clearly the theoretical model is quite capable of predicting the outgassing dimensional changes once the appropriate diffusion coefficient is known, even over the complete thermal cycling environment. 
Why is there such a difference in the diffusion coefficients measured in "space" and in the vacuum chamber? The tests reported show good correlation between "control" and "flight" samples. Moisture saturation and uniform distribution through the laminate was achieved. In addition, both measuring systems correlate very well. The only explanation we have to offer is the possible effect of surface contamination of the LDEF flight samples in the early stages of deployment. Over time, this contamination was removed from the samples due to atomic oxygen. Hence, when the flight samples were tested in the vacuum chamber, no contamination effects were observed. Thus one can account for the apparent increase in outgassing time observed in orbit.

\section{COEFFICIENT OF THERMAL EXPANSION}

As discussed earlier, after outgassing is essentially completed, one finds the thermal-strain response asymptotes, as can be seen in Figure 3 for the $\left[90^{\circ}\right]_{4}$ graphite/epoxy material (5208/T300). This behavior was typical of all our LDEF samples (see ref. 1 for example). Table 2 summarizes the slope values of these curves (i.e., the CTE) for a variety of materials studied. The "ambient" values represent pre-flight measurements which compare in most instances reasonably well with the final asymptotic slopes obtained from the actual flight data. Some postflight CTE results are also presented based on laser interferometer measurements after complete outgassing had occurred.

\section{APPLICATION TO DESIGN}

To demonstrate how this diffusion data and analysis can be used in the design of low distortion laminates, consider the case of a $( \pm \theta)_{s}$ structure. The question being addressed is how much axial distortion can occur in a zero CTE laminate?

Figure 16 presents the variation in the $\alpha_{x}$ and $\alpha_{y}$ CTE values for a $( \pm \theta)_{s}$ laminate fabricated from 5208/T300 material. The curves shown were determined using equation (10). The case of $\alpha_{x}=0$ occurs when $\theta \cong \pm 46^{\circ}$. Using diffusion data to calculate the CME values of $\beta_{x}$ and $\beta_{y}$ from equation (10), one can obtain from Figure 17 a $\beta_{x} \simeq 200 \times 10^{-6} / \% M$ at $\theta=46^{\circ}$.

Assuming a 1-percent moisture uptake prior to launch yields an axial displacement of $\Delta L=$ $200 \times 10^{-6} L$ where $L=$ length of structure. Thus for a $10-\mathrm{m}$ long structure, the axial contraction would be $2.0 \mathrm{~mm}$ for a zero CTE laminate.

\section{CONCLUSIONS}

1. Outgassing produces dimensional changes of polymer matrix composites which asymptote to a constant value once the outgassing process has essentially ceased.

2. A test protocol and analytical model have been formulated that can accurately predict the dimensional changes associated with outgassing as a function of temperature and time in vacuum.

3. Outgassing of the LDEF polymer matrix composites took much longer to asymptote in orbit than in a thermal-vacuum chamber. It is postulated that outgassing caused surface 
contamination of the LDEF samples, thus inhibiting the diffusion process. The surface contaminants were then removed over time by the incident atomic oxygen which proceeded to erode the composite material as well.

4. The analytical model was capable of reproducing the LDEF flight sample response extremely well once a modified diffusion coefficient was used.

5. In general, the asymptotic thermal strain response of the flight samples yielded CTE values close to their original ambient measurements.

\section{ACKNOWLEDGMENTS}

This research was sponsored by the Canadian Space Agency (CSA) under contract 025SR.9F009-1-1435, and two Ontario Centres of Excellence: the Institute for Space and Terrestrial Science and the Ontario Centre for Materials Research. Special thanks are extended to Dr. D.G. Zimcik of the CSA for his continued support of our work.

\section{REFERENCES}

1. Tennyson, R.C.: "Composite Materials in Space-Results from the LDEF Satellite." J. Canadian Aeronautics and Space Institute, vol. 37, No. 3, September 1991.

2. Crank, J., and Park, G.S.: "Diffusion in Polymers.” Academic Press, London, 1968.

3. Shen, Chi-Hung., and Springer, George S.: "Moisture Absorption and Desorption of Composite Materials.” J. Composite Materials, vol. 10, January 1976.

4. Halpin, J.C.: "Primer on Composite Materials: Analysis." Technomic Publishing Company, 1984. 
Table 1. Comparison of flight and control sample diffusion coefficients for [90]4 graphite/epoxy laminates $(5208 / \mathrm{T} 300)$ as measured in vacuum chamber.

\begin{tabular}{|c|c|c|c|c|c|c|}
\hline Sample No. & Status & Temp $\left[{ }^{\circ} \mathrm{C}\right]$ & $M_{i}[\%]$ & $\Delta \varepsilon[\mu \varepsilon]$ & $\begin{array}{c}\text { CME } \\
{[\mu \varepsilon / \text { percent }]}\end{array}$ & $D\left[\mathrm{~mm}^{2} / \mathrm{h}\right]$ \\
\hline 5T5 & Control & 22 & 0.490 & $-1,200$ & 2,449 & 0.00010 \\
\hline 5T5 & Control & 50 & 0.550 & $-1,939$ & 3,525 & 0.00047 \\
\hline 2T13 & Flight & 22 & 0.505 & $-1,212$ & 2,400 & 0.00013 \\
\hline 2T13 & Flight & 22 & 0.510 & $-1,224$ & 2,400 & 0.00008 \\
\hline 2T13 & Flight & 50 & 0.632 & $-1,517$ & 2,400 & 0.00078 \\
\hline 3T6 & Flight & 22 & 0.500 & $-1,200$ & 2,400 & 0.00014 \\
\hline 3T6 & Flight & 22 & 0.510 & $-1,219$ & 2,400 & 0.00009 \\
\hline
\end{tabular}

Table 2. Comparison of CTE data from LDEF experiment AO180.

\begin{tabular}{|c|c|c|c|c|c|}
\hline Sample & Material & $\begin{array}{c}\text { Laminate } \\
\text { Type }\end{array}$ & $\begin{array}{c}\text { Ambient } \\
\text { CTE } \\
{\left[10^{-6 / C]}\right.}\end{array}$ & $\begin{array}{c}\text { Space CTE } \\
{\left[10^{-6 / C}\right]}\end{array}$ & $\begin{array}{c}\text { Postflight* } \\
\text { CTE } \\
{\left[10^{-6} / \mathrm{C}\right]}\end{array}$ \\
\hline Control & T300/5208 & $(90)_{4}$ & - & - & 24.5 \\
Flight & T300/5208 & $(90)_{4}$ & - & - & 24.7 \\
Flight & T300/5208 & $(90)_{4}$ & 28.1 & 28.9 & - \\
Control & T300/5208 & $( \pm 45)_{\mathrm{S}}$ & - & - & 1.93 \\
Flight & T300/5208 & $( \pm 45)_{\mathrm{S}}$ & - & - & -6.53 \\
\hline Flight & T300/934 & $(90)_{4}$ & 26.5 & 27.3 & - \\
\hline Flight & T300/SP-288 & $(90)_{4}$ & 26.3 & 26.8 & - \\
\hline Flight & SP-290 & $( \pm 30)_{\mathrm{S}}$ & 2.8 & 2.21 & - \\
& Boron/Epoxy & $( \pm 60)_{\mathrm{S}}$ & 21.1 & 20.9 & - \\
\hline Flight & SP-328 & $(90)_{4}$ & 61.0 & 59.2 & - \\
& Kevlar/Epoxy & $(0)_{4}$ & 0.18 & 0.83 & - \\
\hline Control & SP-328 & $( \pm 45)_{\mathrm{S}}$ & - & - & -0.04 \\
Flight & SP-328 & $( \pm 45)_{\mathrm{s}}$ & - & - & 2.52 \\
\hline
\end{tabular}

*Measured after complete vacuum outgassing 




Figure 1. Thermal history of LDEF specimen (3T6).



Figure 2. Strain-time history for LDEF specimen (3T6). 


\section{Graphite:Epoxy $\left[90^{\circ}\right]$}

$5208 / \mathrm{T} 300$



Figure 3. Thermal/strain response of LDEF specimen (3T6).

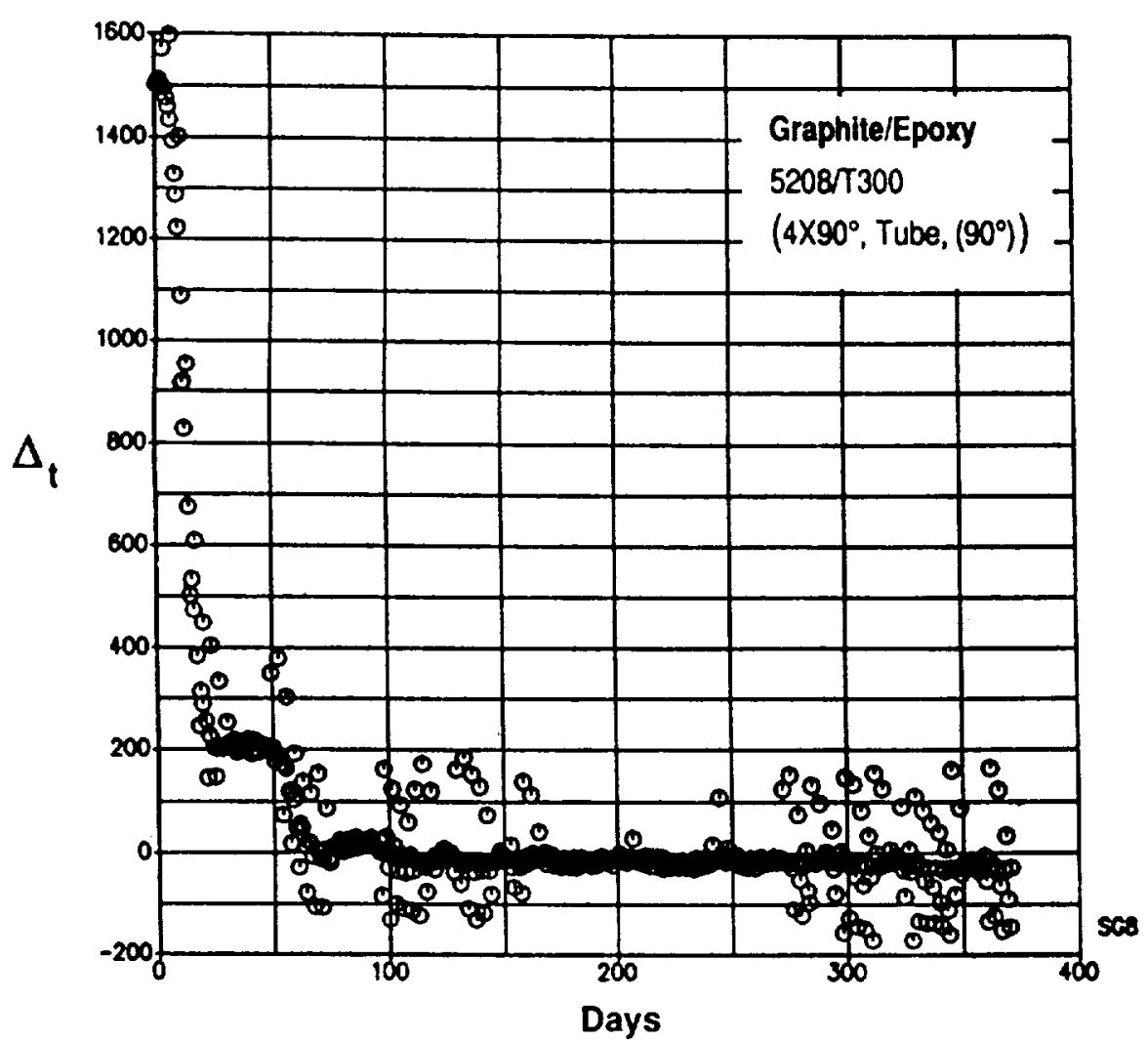

Figure 4. Plot of $\Delta_{t}$ strain versus time in orbit for LDEF specimen (3T6). 


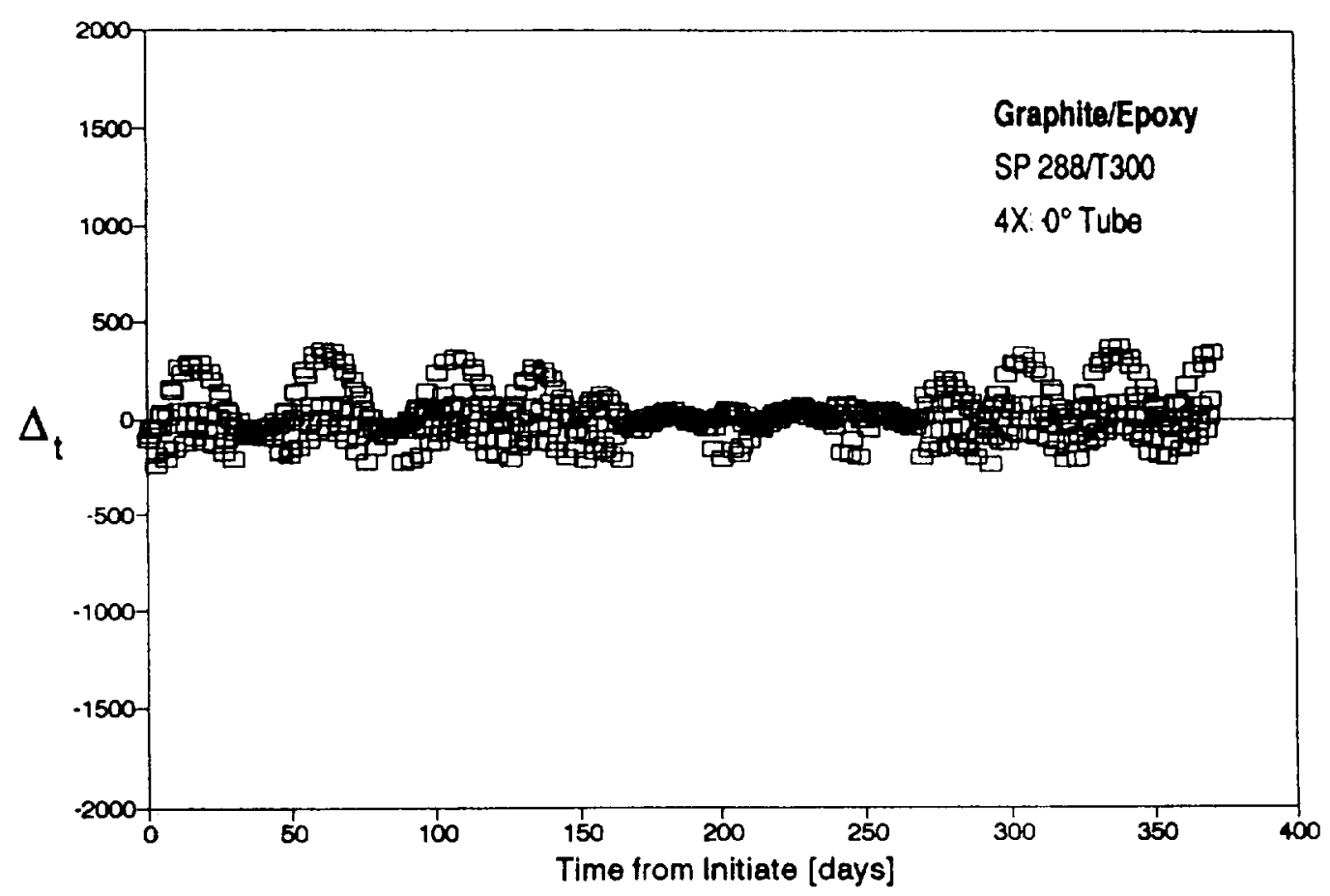

Figure 5. Plot of $\Delta_{t}$ strain versus time in orbit for LDEF specimen.



Figure 6. Comparison of outgassing response measured by strain gauge and laser interferometry. 


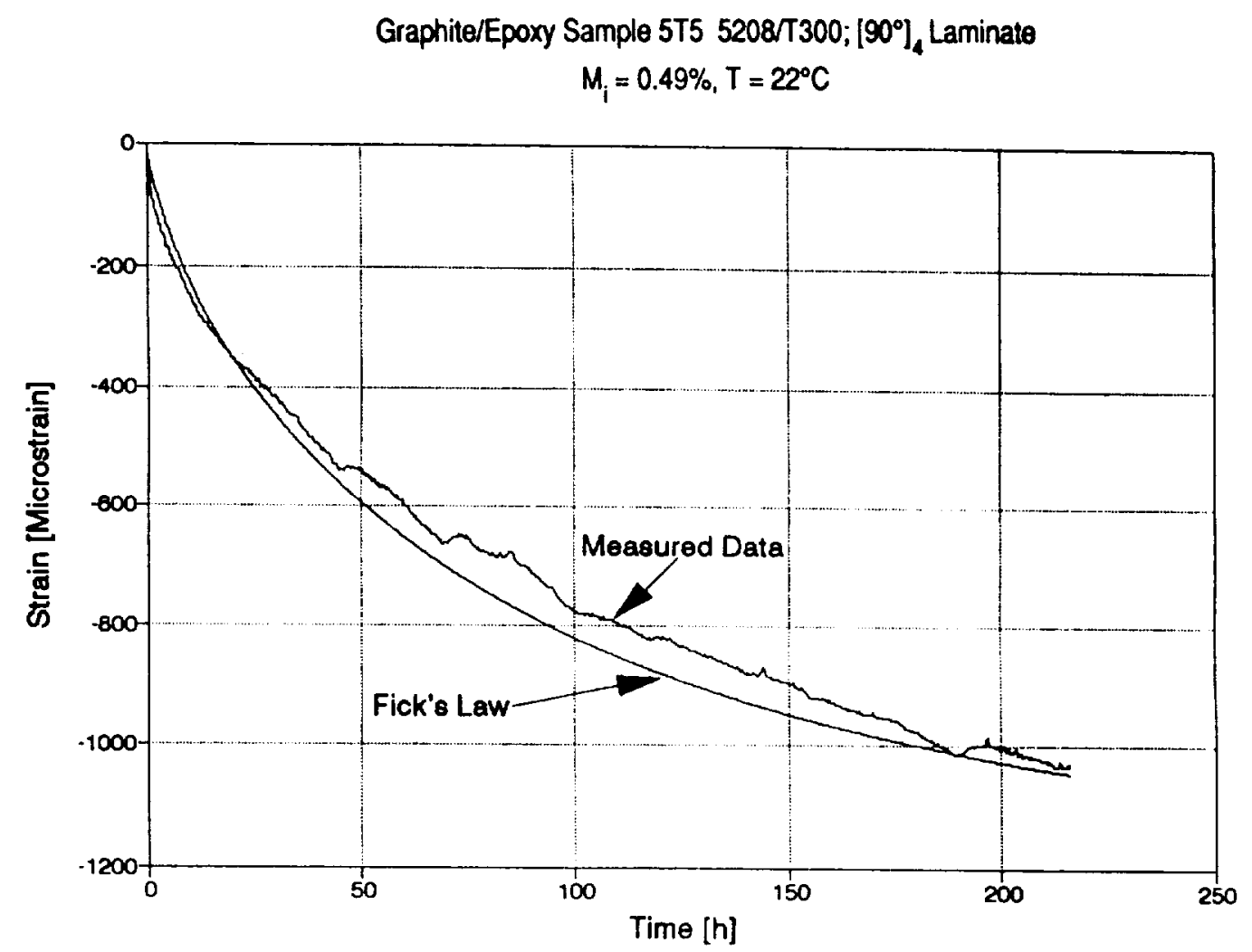

Figure 7. Comparison of outgassing response with Fick's law prediction.

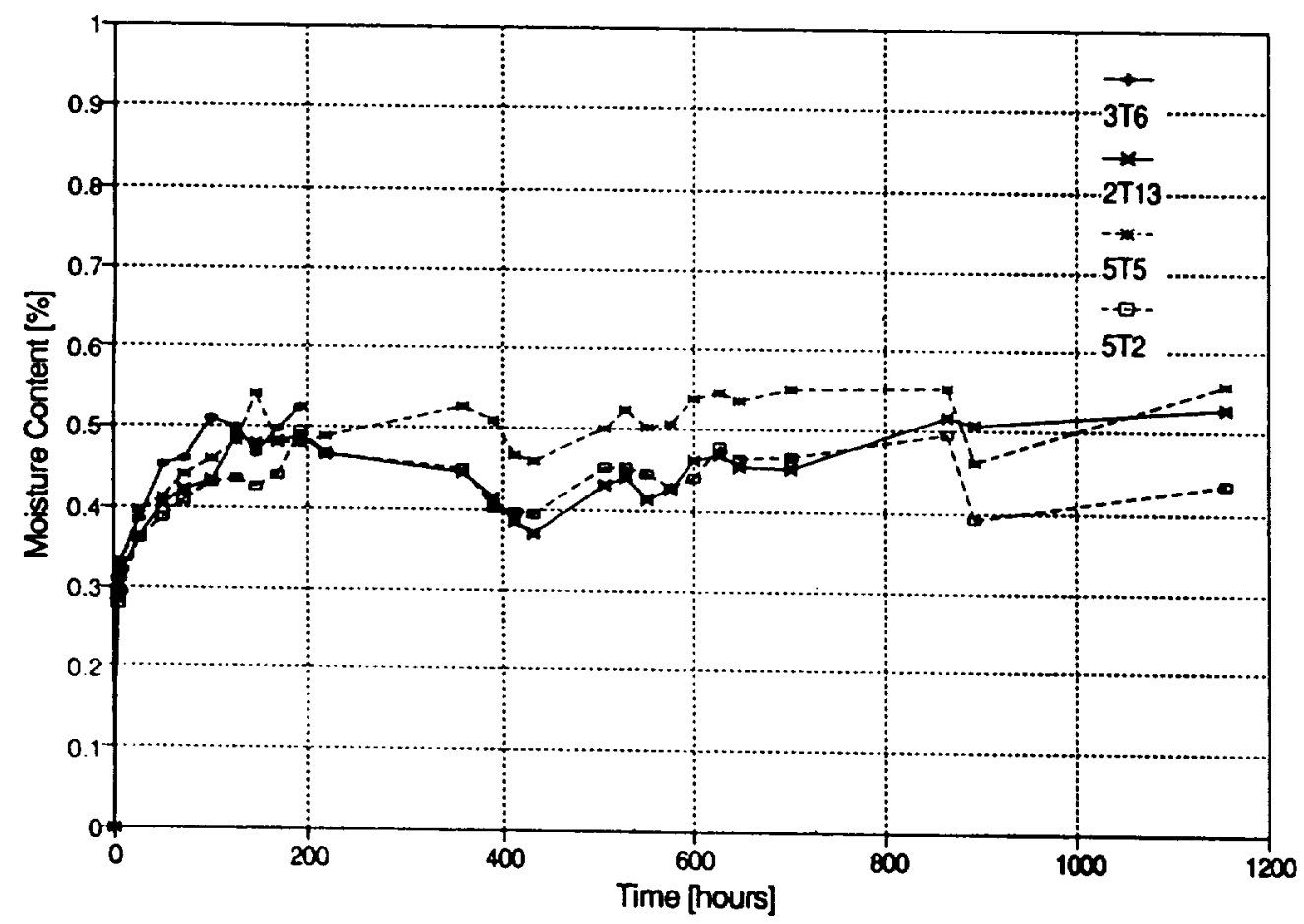

Figure 8. Moisture absorption response of LDEF samples at $50{ }^{\circ} \mathrm{C}$ and 75 -percent RH. 


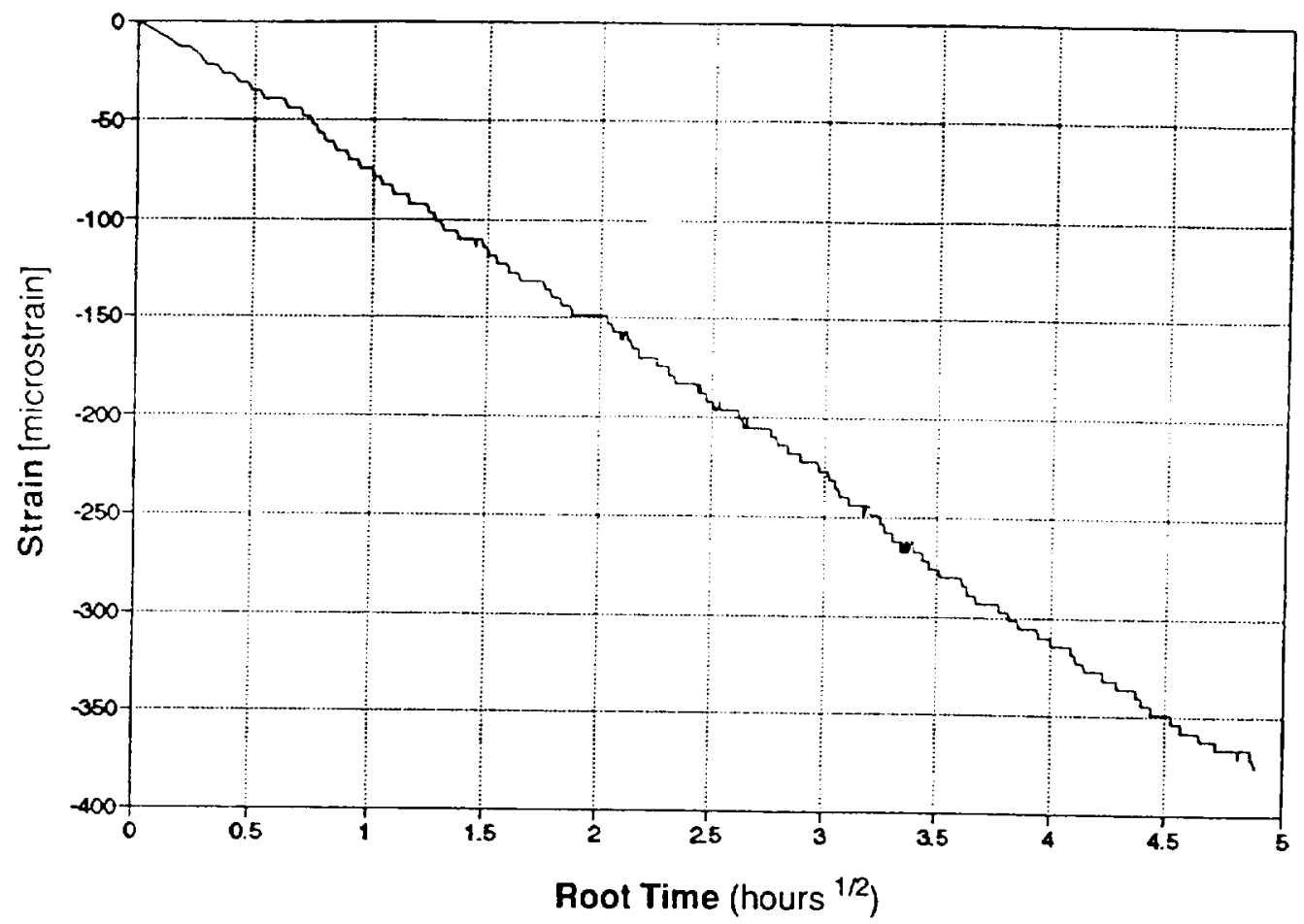

Figure 9. Outgassing test on control specimen (5T5) at $22{ }^{\circ} \mathrm{C}$.

Graphite/Epoxy $\left[90^{\circ}\right]$

$5208 / 7300$

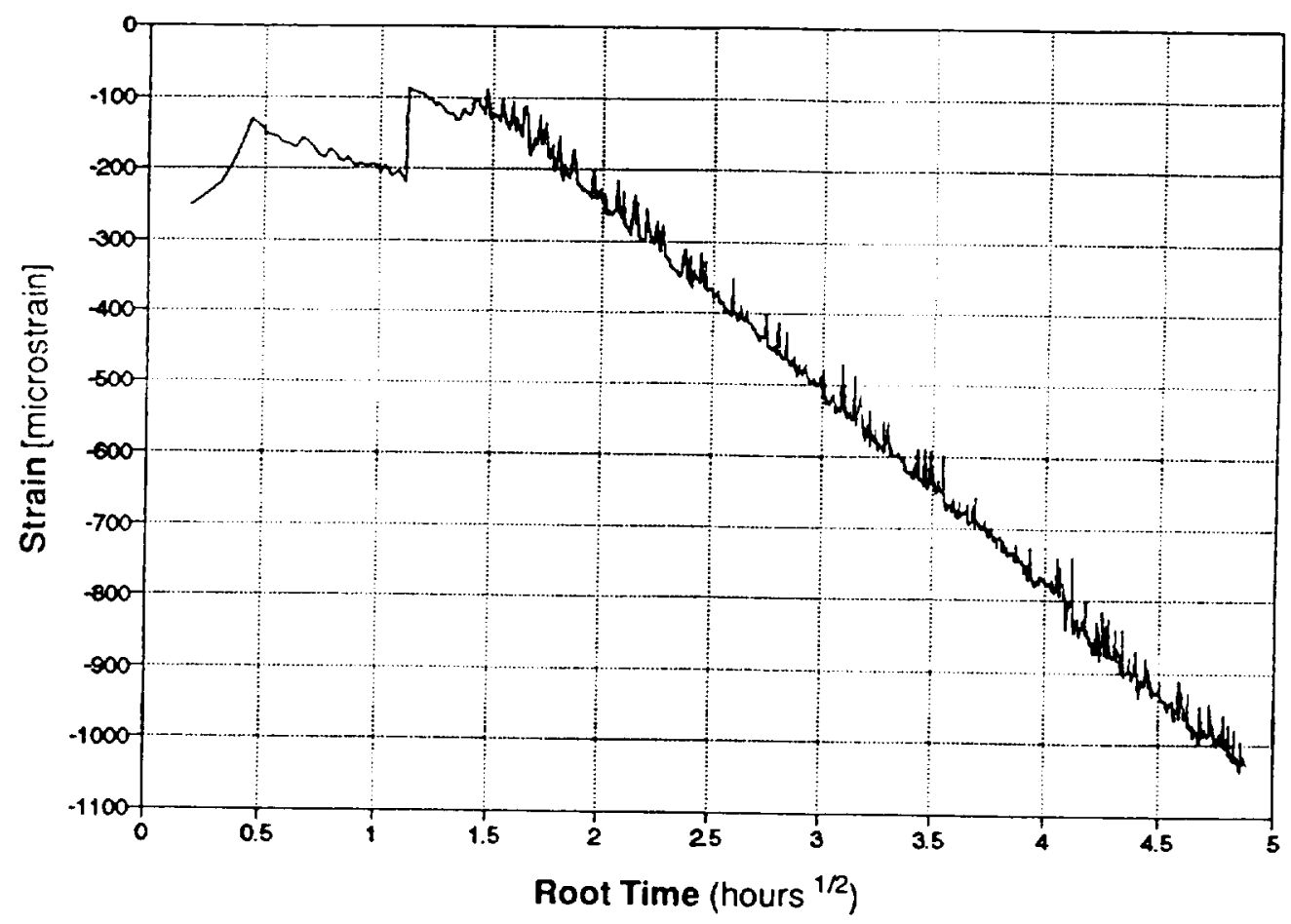

Figure 10. Outgassing test on control specimen $(5 \mathrm{T5})$ at $55^{\circ} \mathrm{C}$. 




Figure 11. Outgassing test on LDEF flight specimen $(2 \mathrm{~T} 13)$ at $22^{\circ} \mathrm{C}$.



Figure 12. Outgassing test on LDEF flight specimen (2T13) at $50^{\circ} \mathrm{C}$. 


\section{Graphite/Epoxy 5208/7300; $\left.190^{\circ}\right]_{4}$}

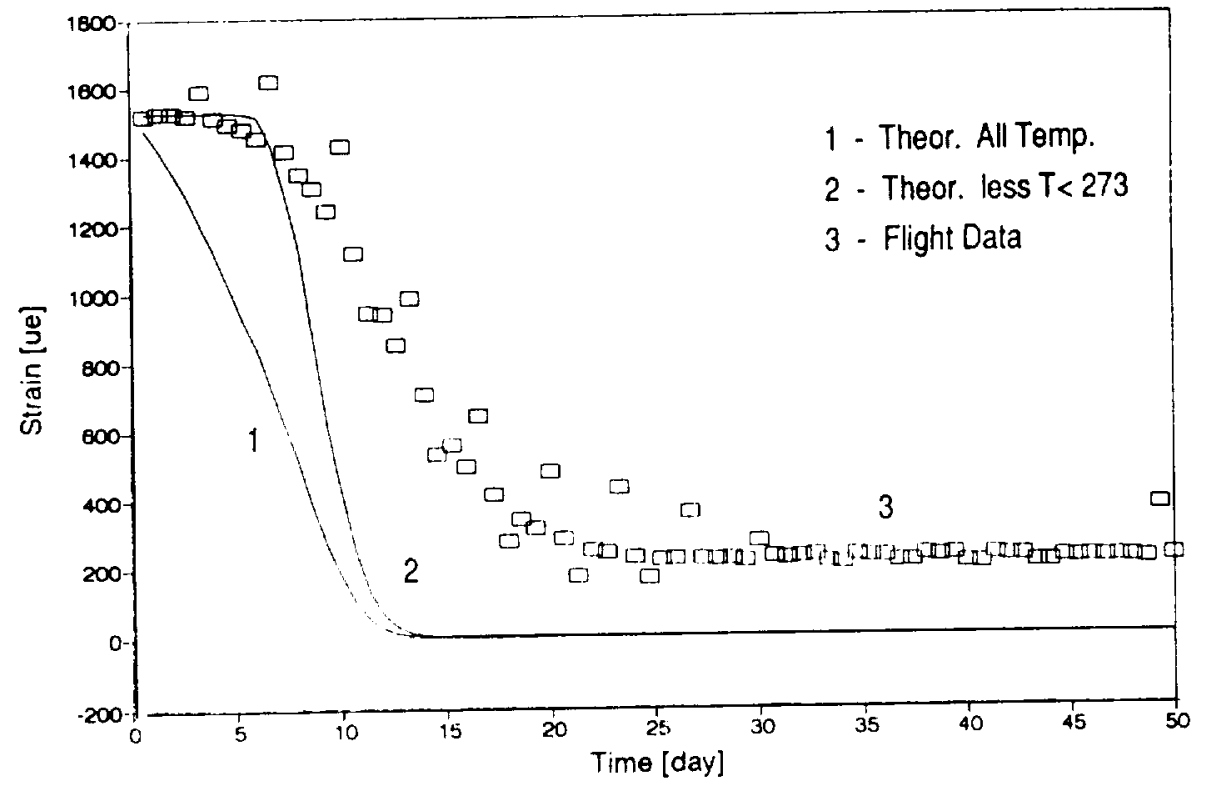

Figure 13. Comparison of predicted outgassing response with LDEF flight sample.

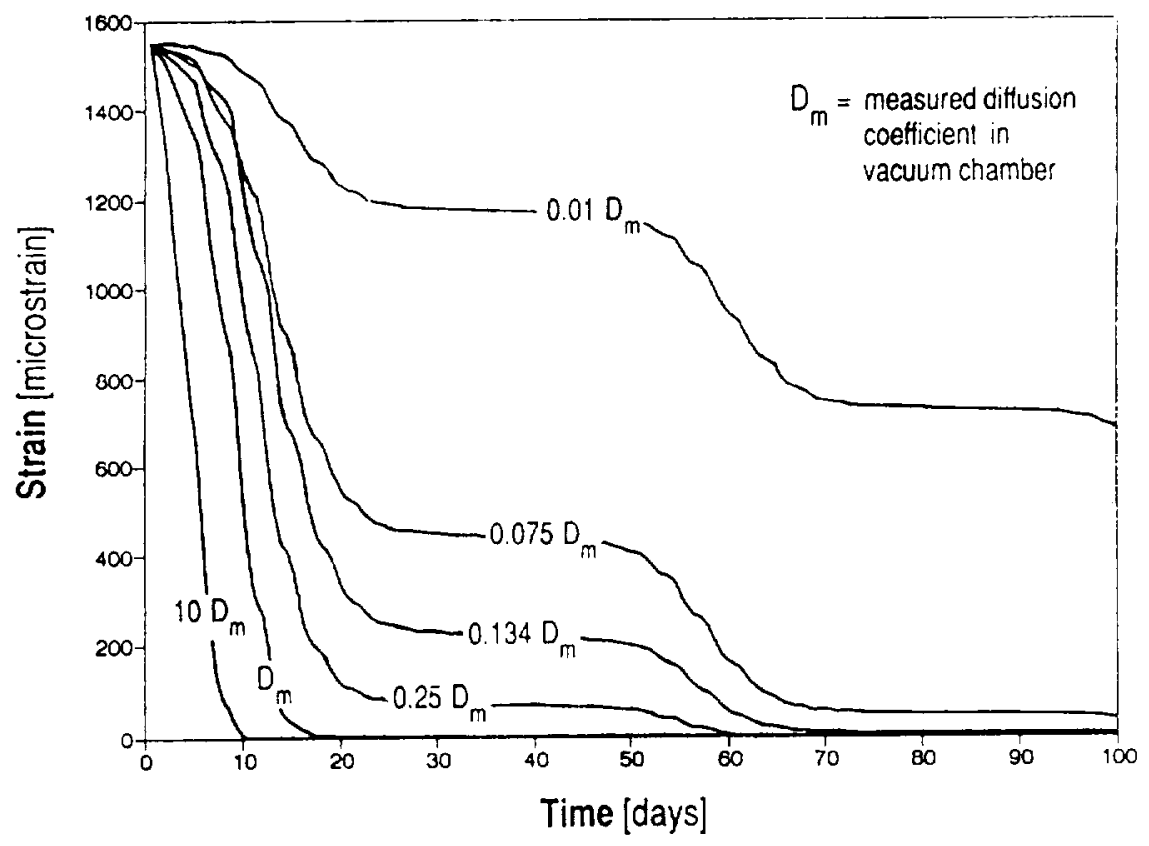

Figure 14. LDEF outgassing predictions for T300/5208 $(90)_{4}$ laminate. 




Figure 15. Comparison of corrected prediction of strain/time response in space with LDEF flight specimen $\left(D^{*}=0.134 D\right)$.

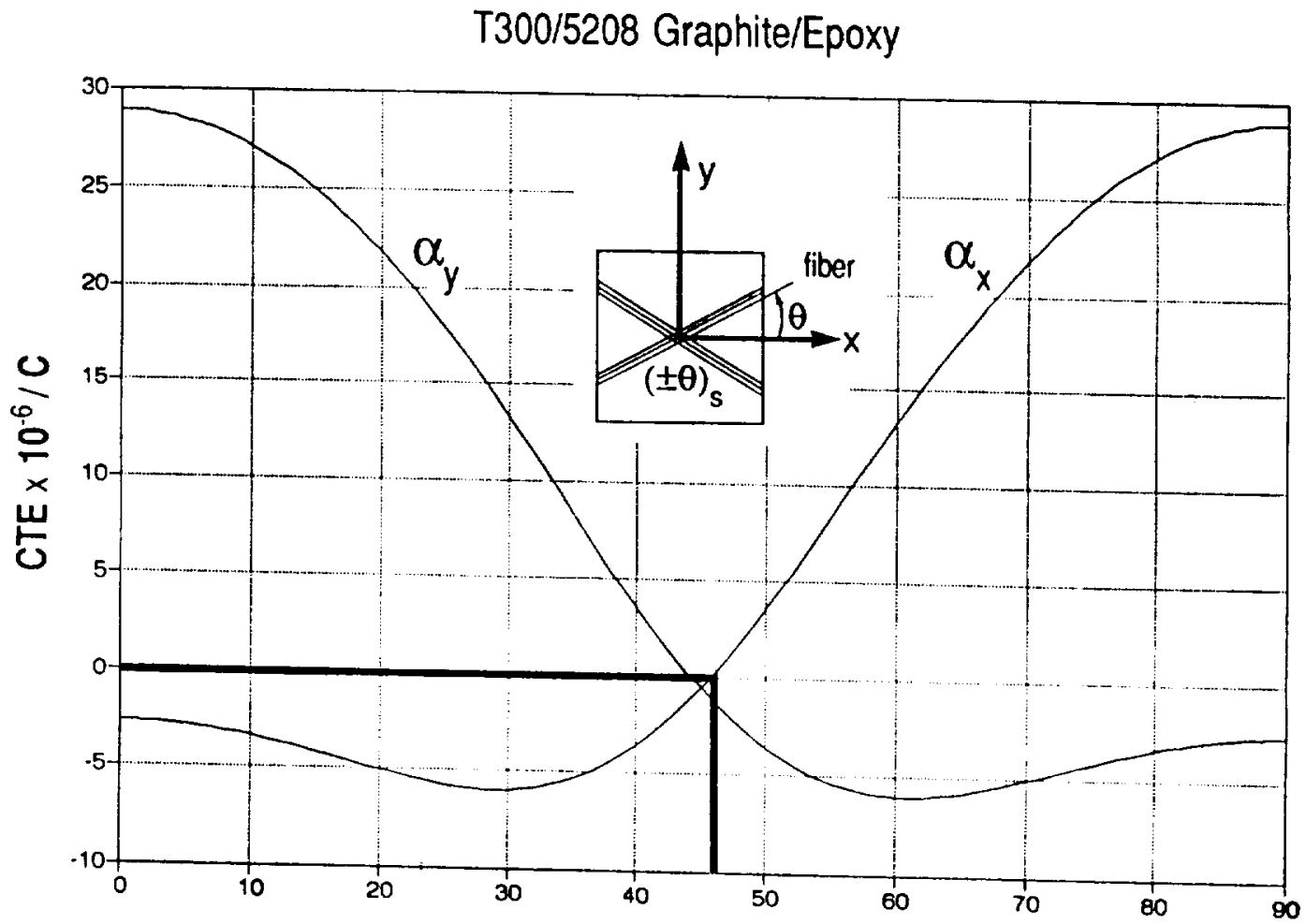

Ply Angle $\left(\theta^{\circ}\right)$

Figure 16. CTE for angle-ply laminates. 
T300/5208 Graphite/Epoxy

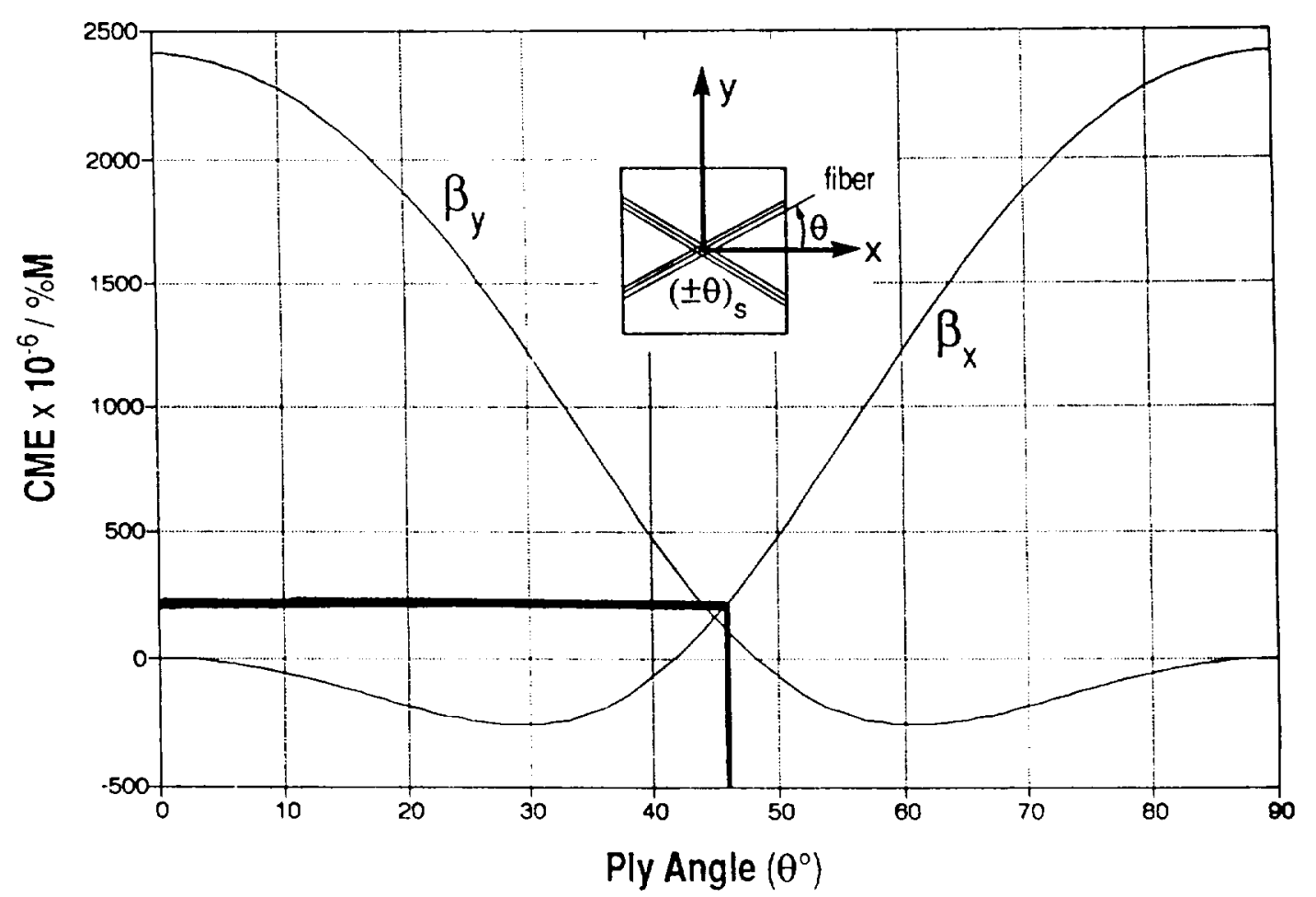

Figure 17. CME for angle-ply laminates. 
1 